\title{
GEODESIC TUBES ON LOCALLY SYMMETRIC SPACES
}

\author{
B. J. PAPANTONIOU* ${ }^{\dagger}$
}

\begin{abstract}
A.bstract. In this paper we state and prove a characteristic relation which exists, between the eigenspaces of the Ricci transformation $R(N,-) N$ acting on the orthocomplement space of $N$ in $T_{m} M$ where $m \in M, M$ being a locally symmetric space, and the Weingarten map $S_{N}$ of small enough geodesic tubes of $M$.
\end{abstract}

\section{Introduction}

It is an interesting problem to see how the properties of geodesic tubes on a Riemannian manifold $(M, g)$ determine the geometry of the ambient space $(M, g)$. This problem has been recently treated in [12] and [13] with $(M, g)$ being a space of constant curvature and a space of constant holomorphic sectional curvature respectively. Similar problems have also been studied earlier, by using the properties of small geodesic spheres, in several papers, see for example [1], [7], [8], [9], [10], [11]. The proofs of the latest papers are based on a significant relation which exists, between the eigenspaces of the Ricci transformation $R(N,-) N$ acting on $\{N\}^{\perp}$ and the Weingarten map $S_{N}$ of small enough geodesic spheres of the space, where $N$ is a unit tangent vector field along a geodesic starting from the centre of the sphere.

In this aspect, we give a corresponding result for geodesic tubes of a locally

Received May 11, 1992.

*1991 Mathematics Subject Classification: 53C05, 53C20, 53C21.

†This work was started at the Univ. of Liverpool and completed at the Michigan State University, where the author was a visiting scholar. 
symmetric space, by using Fermi coordinates, Fermi vector fields and a nice relation which exists between them.

In the second section we give the definitions of Fermi coordinates and Fermi vector fields, some basic properties and the definition of the tubes and tubular hypersurfaces of a Riemannian manifold. In the third section we state and prove our main result in Theorem 3.1.

\section{Preliminaries}

Let $(M, g)$ be an $n$-dimensional connected $C^{\infty}$ Riemannian manifold, $\nabla$ its Riemannian connection and

$$
R_{X Y}=\left[\nabla_{X}, \nabla_{Y}\right]-\nabla_{[X, Y]}
$$

its curvature transformation.

Let $\sigma:(a, b) \rightarrow M ; a, b \in \mathbb{R}$ be a curve of finite length of $M$. To describe the geometry of a Riemannian manifold $M$ in the neighbourhood of a curve $\sigma$ we use Fermi coordinates. E. Fermi introduced these coordinates in [3] and soon utilized by Levi-Civita and L. Eisenhart in the 1920's. We give now some useful definitions and properties, following closely [5] and [6]. To define a system of Fermi coordinates we need, an open neighbourhood of $U=U(\sigma)$ of $\sigma$, for which every point of $U$ can be joined to $\sigma$ by a shortest unit-speed geodesic, meeting $\sigma$ orthogonally and it is assumed that it contains no focal points of $\sigma$. Further, we need an orthonormal frame field $\left\{E_{1}, \ldots, E_{n}\right\}$ along the curve $\sigma$, which may be a geodesic of $M$. Let $m=\sigma(0)$ and $\dot{\sigma}(t)=\left.E_{1}\right|_{\sigma(t)}$.

Definition 2.1. The Fermi coordinates $\left(x_{1}, \ldots, x_{n}\right)$ of $U$ centered at $m=$ $\sigma(0)$, relative to a given orthonormal frame field $\left\{E_{1}, \ldots, E_{n}\right\}$, along the curve $\sigma$ for which $\dot{\sigma}(t)=\left.E_{1}\right|_{\sigma(t)}$, are the real-valued functions defined by

$$
x_{1}\left(\left.\exp _{\sigma(t)} \sum_{j=2}^{n} t_{j} E_{j}\right|_{\sigma(t)}\right)=t
$$




$$
x_{i}\left(\left.\exp _{\sigma(t)} \sum_{j=2}^{n} t_{j} E_{j}\right|_{\sigma(t)}\right)=t_{i}, \quad 2 \leq i \leq n
$$

provided that the numbers $t_{2}, \ldots, t_{n}$ are small enough so that $\exp _{\sigma(t)}$ to be a diffeomorphism.

Since $\exp _{\sigma(t)}$ is a diffeomorphism on $U$ the equations (2.2) and (2.3) define a coordinate system near $m$. Let $\left\{\frac{\partial}{\partial x_{1}}, \ldots, \frac{\partial}{\partial x_{n}}\right\}$ be the coordinate vector fields associated with the Fermi coordinate system $\left(x_{1}, \ldots, x_{n}\right)$.

Lemma 2.1. If $\left(x_{1}, \ldots, x_{n}\right)$ is a system of Fermi coordinates centered at $m \in \sigma$, then the restrictions to $\sigma$ of the coordinate vector fields

$$
\frac{\partial}{\partial x_{2}}, \ldots, \frac{\partial}{\partial x_{n}}
$$

are orthonormal.

Lemma 2.2. Let $\gamma$ be a unit-speed geodesic of $M$ normal to $\sigma$ with $\gamma(0)=$ $m=\sigma(0)$ and let $u=\gamma^{\prime}(0)$. Then there is a system of Fermi coordinates $\left(x_{1}, \ldots, x_{n}\right)$ such that for small $s$ we have:

$$
\left(\frac{\partial}{\partial x_{2}}\right)_{\gamma(s)}=\gamma^{\prime}(s)
$$

and

$$
\left(\frac{\partial}{\partial x_{1}}\right)_{m}=(\dot{\sigma}(t))_{m}, \quad\left(\frac{\partial}{\partial x_{i}}\right)_{m}=[\dot{\sigma}(t)]_{m}^{\perp}, \quad i=2, \cdots, n .
$$

Furthermore,

$$
\left(x_{\alpha} \circ \gamma\right)(s)=s \delta_{\alpha}^{2}
$$

for $1 \leq \alpha \leq n$ where $\delta$ is the Kronecker symbol.

For proofs of these lemmas see for example [5].

Let $\mathcal{X}(U)$ be the Lie algebra of $C^{\infty}$ vector fields on $U$. We introduce a certain finite dimensional Abelian subalgebra of the infinite dimensional Lie algebra. $\mathcal{X}(U)$ 
Definition 2.2. Let $\left(x_{1}, \ldots, x_{n}\right)$ be a Fermi coordinate system of $U=U(\sigma)$ relative to the orthonormal frame field $\left\{E_{1}, \ldots, E_{n}\right\}$. We say that $X \in \mathcal{X}(U)$ is a Fermi vector field relative to $\left(x_{1}, \ldots, x_{n}\right)$ provided

$$
X=\sum_{i=2}^{n} c_{i} \frac{\partial}{\partial x_{i}}
$$

where the $c_{i}$ 's are constants.

We define now two other simple but basic objects, $s$ and $N$ in terms of Fermi coordinates, since they will be needed in the following.

Definition 2.3. Let $\left(x_{1}, \ldots, x_{n}\right)$ be a system of Fermi coordinates for $U=U(\sigma)$. For $s>0$ we put

$$
s^{2}=\sum_{i=2}^{n} x_{i}^{2} \text { and } N=\sum_{i=2}^{n} \frac{x_{i}}{s} \cdot \frac{\partial}{\partial x_{i}} .
$$

For $m \in \sigma$ it is easily proved that the definitions of $s$ and $N$ are independent of the choice of Fermi coordinates at $m$. In fact for $m^{\prime} \in M$ near $\sigma, s\left(m^{\prime}\right)=d\left(m^{\prime}, \sigma\right)$ where $d$ is the distance function of $M$. Furthermore,

$$
N_{\gamma(s)}=\left(\frac{\partial}{\partial x_{2}}\right)_{\gamma(s)}=\gamma^{\prime}(s), \quad s>0
$$

where $\gamma$ is the unique geodesic from $m^{\prime}$ to $\sigma$ which meets $\sigma$ orthogonally at $m=\gamma(0)$.

In what follows we assume that $\sigma$ is also a geodesic of $M$ and put $A=\frac{\partial}{\partial x_{1}}$. The most importance properties of $s$ and $N$ and Fermi vector fields are included in the following:

Lemma 2.3. [5]. Let $X$ be a Fermi vector field for $U=U(\sigma)$ and $A, N, s$ as previously. Then we have:
1. $\nabla_{N} N=0$
4. $A(s)=0$
7. $[N, s X]=X(s) N$
2. $g(N, N)=1$
5. $[X, A]=[N, A]=0$
3. $N(s)=1$
8. $\nabla_{N}^{2} Z=R(N, Z) N$
6. $[N, X]=-\frac{1}{s} X+\frac{1}{s} X(s) N$ 
for any $Z=A+s X$.

If $\gamma$ is curve and $Y$ is a vector field along $\gamma$, write $Y^{\prime}=\nabla_{\gamma^{\prime}} Y$ and $Y^{\prime \prime}=$ $\nabla_{r}^{2}, Y$. Then we have,

Definition 2.4. A vector field $Y$ along a geodesic $\gamma$ is called a Jacobi field if it satisfies the following second order differential equation:

$$
Y^{\prime \prime}=R\left(\gamma^{\prime}, Y\right) \gamma^{\prime}
$$

It is now understood that the vector field $Z$, in relation (8) of Lemma 2.3 , is a Jacobi vector field. Moreover, if $\gamma$ is a geodesic normal to $\sigma$ at $m=\sigma(0)$ and $X$ is a Fermi vector field on $U=U(\sigma)$, then the restrictions to $\gamma$ of $A$ and $s X$ are also Jacobi vector fields, as one easily concludes it from the relation (8) of Lemma, 2.3.

Therefore, we come to the following.

Lemma 2.4. Let $X_{i}^{*}=\left(\frac{\partial}{\partial x_{i}}\right), i=1 ; 3, \cdots, n$ be the coordinate Fermi fields relative to the Fermi coordinate system $\left(x_{1}, \ldots, x_{n}\right)$ of $U=U(\sigma)$, then the fields

$$
\begin{aligned}
& Y_{1}(s)=X_{1}^{*}(s)=\left(\frac{\partial}{\partial x_{1}}\right)_{\gamma(s)}, \\
& Y_{3}(s)=s X_{3}^{*}(s)=s\left(\frac{\partial}{\partial x_{3}}\right)_{\gamma(s)}, \ldots, Y_{n}(s)=s X_{n}^{*}(s)=s\left(\frac{\partial}{\partial x_{n}}\right)_{\gamma(s)}
\end{aligned}
$$

are Jacobi vector fields along the geodesic $\gamma$.

Let $\sigma:(a, b) \rightarrow M$ be a curve of finite length ( $\sigma$ may also be a geodesic), of a Riemannian manifold $(M, g)$. We give now the definition of a tube about the curve $\sigma$.

Definition 2.5. A (solid) tube of radius $s \geq 0$ about a curve $\sigma$ is the set of points of $M$ given by

$$
T(\sigma, s)=\left\{\exp _{\sigma(t)} X \mid X \in M_{\sigma(t)}, g(X, X)=1, g(X, \dot{\sigma}(t))=0, a<t<b\right\}
$$

where $M_{\sigma(t)}$ denotes the tangent space of $M$ at the point $\sigma(t)$. 
For small $s>0$, we call the hypersurface of the form

$$
P_{s}=\left\{m^{\prime} \in T(\sigma, s) / d\left(m^{\prime}, \sigma\right)=s\right\}
$$

the tubular hypersurface at distance $s$ form $\sigma$, or just Tube. If $\sigma$ is a geodesic of $M$, then the corresponding tubes are called geodesic tubes. The vector field $N$ now is the unit normal to each of the tubular hypersurfaces $s=$ const., about the curve $\sigma$ of $M$.

\section{Geodesic Tubes and the Main Result}

Let $(M, g)$ be a Riemannian manifold of dimension $n>2$ and let $U$ be an open neighbourhood of a point $m$ in $M$. Let $\sigma$ be a geodesic of $M$ and $P_{s}$ the geodesic tube of radius $s$ about $\sigma$ contained in the open neighbourhood $U$. We always assume that the radius $s$ of $P_{s}$ is less than the distance of $\sigma$ to its nearest focal point. Assume that $m=\sigma(0)$. Let $m^{\prime} \in P_{s}$ and $\gamma=\gamma(s)$ be the geodesic of $M$ containing $m^{\prime}$ belonging in $U$ and meeting $\sigma$ orthogonally at $m=\sigma(0)$. Suppose that $\gamma:(-r, r) \rightarrow U$, is parametrized by its arc length and $\gamma(0)=m$. Choose an orthonormal basis $\left\{E_{1}, \ldots, E_{n}\right\}$ for the tangent space $M_{m}$ such that $E_{1}=\sigma^{\prime}(0), E_{2}=\gamma^{\prime}(0)$ and let $\left\{x_{i}\right\}, i=1, \cdots, n$ be the corresponding Fermi coordinate system on $P_{s}$. The unit tangent vector field to geodesic rays from $m$ on $U-\sigma$ is then $N$ given by (2.9), where $s$ denotes the geodesic distance from $m$. Choose the nonzero vector $W_{m}=\sum_{i=3}^{n} a_{i}\left(\partial / \partial x_{i}\right)_{m}$ normal to $E_{2}=N_{m}$. Let $X_{1}=\frac{\partial}{\partial x_{1}}$ and $X_{2}=\sum_{i=3}^{n} a_{i} s\left(\partial / \partial x_{i}\right)$ on $U$.

Lemma 3.1. On $\gamma-\{m\}$, we have:

$$
\text { i) }\left[X_{i}, N\right]=0, \quad \text { ii) } R\left(N, X_{i}\right) N=\nabla_{N}^{2} X_{i}, \quad i=1,2
$$

Proof. The proof of (ii) is a consequence of Lemma 2.3 , so we will only prove that $\left[X_{2}, N\right]=0$. By Lemma 2.3 relation (7) we get

$$
\left[N, X_{2}\right]=\left[N, \sum_{i=3}^{n} a_{i} s\left(\partial / \partial x_{i}\right)\right]=\left(\sum_{i=3}^{n} a_{i}\left(\frac{\partial s}{\partial x_{i}}\right)\right) N
$$


but since

$$
s^{2}=\sum_{i=2}^{n} x_{i}^{2}
$$

we get

$$
\frac{\partial s}{\partial x_{i}}=\frac{x_{i}}{s}
$$

Therefore,

$$
\left[N, X_{2}\right]=\left(\sum_{i=3}^{n} \frac{a_{i} x_{i}}{s}\right) N
$$

But on $\gamma-\{m\}$, by the definition of Fermi coordinates $\sum_{i=3}^{n} a_{i} x_{i}=0$. Hence we proved that

$$
\left[N, X_{2}\right]=0
$$

From this Lemma one immediately concludes that

$$
\nabla_{X_{i}} N=\nabla_{N} X_{i}, \quad i=1,2
$$

on $\gamma-\{m\}$.

Consider, now, the vector fields $Y_{i}, i=1,2$ on $\gamma$ defined by

$$
\left.Y_{1}\right|_{\gamma(\sigma)}=\left(\frac{\partial}{\partial x_{1}}\right)_{\gamma(\sigma)},\left.Y_{2}\right|_{\gamma(\sigma)}=\sum_{i=3}^{n} a_{i} \sigma\left(\frac{\partial}{\partial x_{i}}\right)_{\gamma(\sigma)},-r<\sigma<r
$$

As a consequence of Lemma 3.1 we will have:

$$
\nabla_{Y_{i}} N=\nabla_{N} Y_{i}, \quad i=1,2
$$

on $\gamma-\{m\}$, and, by continuity,

$$
R\left(N, Y_{i}\right) N=\nabla_{N}^{2} Y_{i} \quad \text { on } \quad \gamma, \quad i=1,2
$$

where $N=e_{2}$ and $\left\{e_{1}, \ldots, e_{n}\right\}$ is the parallel translation of $\left\{E_{1}, \ldots, E_{n}\right\}$ along $\gamma$

From the above analysis it is clear that $Y_{i}, i=1,2$ are Jacobi vector fields along $\gamma$ for which

$$
\text { (I) }\left\{\begin{array} { l } 
{ Y _ { 1 } ( 0 ) = E _ { 1 } } \\
{ Y _ { 1 } ^ { \prime } ( 0 ) = 0 }
\end{array} , \quad \text { (II) } \left\{\begin{array}{l}
Y_{2}(0)=0 \\
Y_{2}^{\prime}(0)=W_{m}
\end{array}\right.\right.
$$


In particular $Y_{i}, i=1,2$ are normal to $e_{2}=N$ and for any point $q$ on $\gamma$ the subspace of $M_{q}$ normal to $\left(e_{2}\right)_{q}$ is formed by evaluation all such Jacobi vector fields at $q$.

Write now $S_{N}=-\nabla N$. For any geodesic tube $P_{s}$ in $U$ about $\sigma$, the restriction of $S_{N}$ to tangent vectors to $P_{s}$ is just the Veingarten map with respect to $N$ as unit normal vector field.

Proposition 3.1. We have:

$$
\begin{aligned}
& \text { i) } S_{N} Y_{i}=-\nabla_{N} Y_{i} \\
& \text { ii) } R\left(N, Y_{i}\right) N=S_{N}^{2} Y_{i}-\left(\nabla_{N} S_{N}\right) Y_{i}, \quad i=1,2
\end{aligned}
$$

for all vector fields $Y_{i}, i=1,2$ orthogonal to $N$, along $\gamma$.

\section{Proof.}

i) From the definition of the Weingarten map (shape operator) and the relation (3.4) we have:

$$
S_{N} Y_{i}=-\nabla_{Y_{i}} N=-\nabla_{N} Y_{i}, \quad i=1,2 .
$$

ii) First, let $Y_{i}$ be a Jacobi vector field as above, then along $\gamma-\{m\}$ we have:

$$
\begin{aligned}
R\left(N, Y_{i}\right) N & =\nabla_{N} \nabla_{Y_{i}} N=-\nabla_{N}\left(S_{N} Y_{i}\right)=-S_{N} \nabla_{N} Y_{i}-\left(\nabla_{N} S_{N}\right) Y_{i} \\
& =-S_{N} \nabla_{Y_{i}} N-\left(\nabla_{N} S_{N}\right) Y_{i}=S_{N}^{2} Y_{i}-\left(\nabla_{N} S_{N}\right) Y_{i} .
\end{aligned}
$$

Now, since $R(N,-) N, S_{N}^{2}$ and $\nabla_{N} S_{N}$ are tensorial, from the above remarks it is valid for arbitrary vector fields $Y$ on $U-\sigma$, where we note from the definition of $S_{N}$ that

$$
S_{N} N=-\nabla_{N} N=0
$$

By continuity now the result is valid at $m$.

We state and prove now the main result.

Theorem 3.1. Let $m$ be a point in a Riemannian loally symmetric space $M$ of dimension $n>2$. Then $m$ has a neighbourhood $U$ such that, for each 
unit vector $N_{m} \in M_{m}$ and corresponding geodesic $\gamma$, the parallel translate of an eigenspace of the linear map $R\left(N_{m},-\right) N_{m}$ along $\gamma$, is contained in an eigenspace of the Weingarten map $S_{N}$, for each geodesic tube in $U$ about a given geodesic $\sigma$, passing from $m$ and meeting $\gamma$ orthogonally at $m$.

Proof. Let $M$ be a Riemannian locally symmetric space and $m \in M$. Following the same notation, as previously, suppose that $E_{1}$ and $W_{m}$ satisfy

$$
R\left(N_{m}, E_{1}\right) N_{m}=k E_{1} \text { and } R\left(N_{m}, W_{m}\right)=k W_{m}, k \in R \text {. }
$$

Since $M$ is locally symmetric $\nabla R=0$, or $R(N,-) N$ is parallel along $\gamma$, hence

$$
R\left(N, \frac{\partial}{\partial x_{1}}\right) N=k \frac{\partial}{\partial x_{1}} \text { and } R(N, W) N=k W
$$

Let $Y_{i}, i=1,2$ be the Jacobi vector ficlds on $\gamma$ satisfying (3.5).

Consider the vector field $f_{1} \frac{\partial}{\partial x_{1}}$. We are interested for those functions $f_{1}$ for which this vector field is a Jacobi vector field along $\gamma$, with the same initial conditions (3.5-I) as $Y_{1}$. So, we will have:

$$
\begin{aligned}
& \left(f_{1} \frac{\partial}{\partial x_{1}}\right)_{m}=E_{1}, \quad \text { or } \quad f_{1}(0)=1 \\
& \left(f_{1} \frac{\partial}{\partial x_{1}}\right)_{m}^{\prime}=0, \quad \text { or } \quad f_{1}^{\prime}(0)=0 .
\end{aligned}
$$

Moreover,

$$
R\left(N, f_{1} \frac{\partial}{\partial x_{1}}\right) N=f_{1} R\left(N, \frac{\partial}{\partial x_{1}}\right) N=k f_{1} \frac{\partial}{\partial x_{1}}
$$

and

$$
\nabla_{N}^{2}\left(f_{1} \frac{\partial}{\partial x_{1}}\right)=f_{1}^{\prime \prime} \frac{\partial}{\partial x_{1}}
$$

Hence,

$$
f_{1}^{\prime \prime}=k \cdot f_{1}
$$

with initial conditions (3.11).

It is now easy, this equation to be solved explicitly and get

$$
f_{1}(\sigma)=\left\{\begin{array}{lll}
\cosh (\sqrt{k} \sigma), & \text { if } & k>0 \\
\cos (\sqrt{|k|} \sigma), & \text { if } & k<0 \\
1, & \text { if } & k=0
\end{array}\right.
$$


Therefore, when $\frac{\partial}{\partial x_{1}}$ is an eigenvector field of $R(N,-) N$, corresponding to the eigenvalue $k$, then $f_{1} \cdot \frac{\partial}{\partial x_{1}}$ is a Jacobi vector field on $\gamma$ when we choose $f_{1}$ given by (3.13).

Next, we are interested for those functions $f_{2}$, for which $f_{2} W$ is also a Jacobi vector field along $\gamma$, with the same initial conditions (3.5-II), as $Y_{2}$. So,

$$
\begin{array}{lll}
\left(f_{2} W\right)_{m}=0 & \text { or } & f_{2}(0)=0 \\
\left(f_{2} W\right)_{m}^{\prime}=W_{m} & \text { or } & f_{2}^{\prime}(0)=1
\end{array}
$$

But, as previously, we have:

$$
R\left(N, f_{2} W\right) N=k f_{2} W \text { and } \nabla_{N}^{2}\left(f_{2} W\right)=f_{2}^{\prime \prime} W
$$

from which

$$
f_{2}^{\prime \prime}=k f_{2},
$$

with initial conditions (3.14). So, we have:

$$
f_{2}(\sigma)=\left\{\begin{array}{lll}
\frac{1}{\sqrt{k}} \sin h(\sqrt{k} \sigma), & \text { if } & k>0 \\
\frac{1}{\sqrt{|k|}} \sin (\sqrt{|k|} \sigma), & \text { if } & k<0 \\
\sigma, & \text { if } & k=0
\end{array}\right.
$$

Thus, when $W$ is an eigenvector field of $R(N,-) N$ corresponding to the eigenvalue $k$, then $f_{2} W$ is a Jacobi vector field on $\gamma$ when we choose $f_{2}$ given by (3.17).

Therefore, we found that

$$
Y_{1}=f_{1} \frac{\partial}{\partial x_{1}}, \quad Y_{2}=f_{2} W
$$

As a consequence now of (3.4) and the definition of $S_{N}$ we have:

$$
S_{N}\left(\frac{\partial}{\partial x_{1}}\right)=-\nabla_{\frac{\partial}{\partial x_{1}}} N=-\nabla_{\frac{1}{f_{1}} Y_{1}} N=-\frac{1}{f_{1}} \cdot \nabla_{Y_{1}} N=-\frac{1}{f_{1}} \nabla_{N} Y_{1} .
$$

But

$$
\nabla_{N} Y_{1}=\nabla_{N}\left(f_{1} \frac{\partial}{\partial x_{1}}\right)=\left(\nabla_{N} f_{1}\right) \frac{\partial}{\partial x_{1}}=N\left(f_{1}\right) \frac{\partial}{\partial x_{1}}
$$


so,

$$
S_{N}\left(\frac{\partial}{\partial x_{1}}\right)=-\frac{N\left(f_{1}\right)}{f_{1}} \cdot \frac{\partial}{\partial x_{1}}
$$

or, using (3.13) we get equivalently

$$
S_{N}\left(\frac{\partial}{\partial x_{1}}\right)=\left\{\begin{array}{lll}
-\sqrt{k}(\tan h \sqrt{k} \sigma) \frac{\partial}{\partial x_{1}}, & \text { if } & k>0 \\
\sqrt{|k|}(\tan \sqrt{|k|} \sigma) \frac{\partial}{\partial x_{1}}, & \text { if } & k<0 \\
0, & \text { if } & k=0
\end{array}\right.
$$

Similarly, we have

$$
\begin{aligned}
S_{N} W & =-\nabla_{W} N=-\nabla_{\frac{1}{f_{2}} Y_{2}} N=-\frac{1}{f_{2}} \nabla_{Y_{2}} N=-\frac{1}{f_{2}} \nabla_{N} Y_{2} \\
& =-\frac{1}{f_{2}} \cdot \nabla_{N}\left(f_{2} W\right)=-\frac{1}{f_{2}} N\left(f_{2}\right) W
\end{aligned}
$$

so,

$$
S_{N} W=-\frac{N\left(f_{2}\right)}{f_{2}} \cdot W
$$

or using (3.17) we equivalently get:

$$
S_{N} W=\left\{\begin{array}{lll}
-\sqrt{k}(\cot h \sqrt{k} \sigma) W, & \text { if } & k>0 \\
-\sqrt{|k|}(\cot \sqrt{|k|} \sigma) W, & \text { if } & k<0 \\
-\frac{1}{\sigma} W, & \text { if } & k=0
\end{array}\right.
$$

Since now the sectional curvature at $m$ is bounded, the set of eigenvalues $k$ of $R\left(N_{m},-\right) N_{m}$ taken over all unit vectors $N_{m}$ is bounded, say $|k|\left\langle\lambda^{2}, \lambda\right\rangle 0$. Thus if $U$ is a tubular neighbourhood of radius $<\pi / \lambda$, then $f_{i}, i=1,2$ is nowhere zero on $\gamma-\{m\}$. Therefore, as a consequence, we get the required result from (3.19) and (3.21) or equivalently, from (3.20) and (3.22).

In [12] and [13] we recently characterized spaces of constant sectional curvature by using the shape operator of small enough geodesic tubes.

It seems to me now, after this result, that locally symmetric spaces of higher rank, may be also characterized, by using the properties of small geodesic tubes.

\section{Acknowledgement}

The author expresses his appreciation and thanks to Dr. A. J. Ledger for many valuable conversations. 


\section{References}

[1] D. E. Blair and A. J. Ledger, "A characterization of oriented Grassman manifolds", Rocky Mountain Journal of Math., 14, No. 3, (1984), 573-584.

[2] B. Y. Chen, "Geometry of submanifolds", Vol. 22, M. Dekker (1973).

[3] E. Fermi, "Sopra $i$ fenomeni che arrengono in vicinanza di una linea oraria", Atti $R$. Acad. Lincei Rend. cl. Sci. Fis. Mat. Natur., 31 (1992), 21-23, 51-52, 101-103.

[4] A. Gray, "Comparison theorems for volumes of tubes as generalizations of the Weyl tube formula", Topology, 21 (1982), 201-228.

[5] A. Gray, "Tubes", Addision Wesley Publ. Company, (1990).

[6] A. Gray and L. Vanhecke, "The volumes of tubes about curves in a Riemannian manifold", Proc. Lond. Math. Soc., XLIV, (1982), 215-243.

[7] A. J. Ledger, "A characterization of complex Grassmann manifolds", Indian J. Pure Appl. Math., 15 (1) (1984), 99-112.

[8] A. J. Ledger, "Geodesic spheres on Grassmann manifolds", Yokohama Math. J., 34 (1986), 59-71.

[9] B. J. Papantoniou, "An investigation of a tensor field on the Grassmannian $G_{2, p}(\mathbb{R})$ ", Indian J. Pure Appl. Math., 16 (10) (1986), 1104-1116.

[10] B. J. Papantoniou, "A characterization of the symmetric space $S U(n) / S O(n)$ by geodesic spheres", Linear Algebra and its Applications, 136 (1990), 133-164.

[11] B. J. Papantoniou, "A characterization of the Grassmann manifold $G_{2, p}(\mathbb{R})$ ", another review, Internat. J. Math. and Math. Sci., Vol. 12 No. 2 (1989), 321-332.

[12] B. J. Papantoniou, "Geodesic tubes and spaces of constant curvature", submitted.

[13] B. J. Papantoniou, "Geodesic Tubes, Jacobi Vector Fields and Kähler Manifolds", submitted.

[14] L. Vanhecke and T. Willmore, "Jacobi fields and geodesic spheres", Proc. of the Royal Soc. of Edinburgh, 82A (1979), 233-240.

[15] H. Weyl, "On the volume of tubes", Amer. J. Math., 61 (1939), 461-472.

University of Patras, Department of Mathematics, 26110, Patras, Greece. 